\title{
The Cytoplasmic Droplets of Rat Epididymal Spermatozoa Show Diminution of Glycosyl Transferases During Spermatozoa Epididymal Maturation
}

Peter Chan, M.Sc., M.D.C.M.

To whom correspondence should be addressed:

Department of Urology

McGill University

Montreal, QC

Canada H3A 1A1

\begin{abstract}
The cytoplasmic droplet of the epididymal spermatozoon is a small localized outpouching of cytoplasm of the tail and is of unknown significance. Electron microscopy revealed flattened saccular elements of almost exclusive membranous components. In this study, a simple, reproducible method for the isolation of saccular elements from the caput and the cauda of rat epididymal spermatozoa is presented. Using exogenous glycosylation assays and immunocytochemistry for light and electron microscopy, we confirm the presence of three Golgi markers in the saccular elements of rat cytoplasmic droplets, namely, a-2,6-sialyltransferase, b1,4-galactosyltransferase and TGN-38 (trans-Golgi network protein). The variation in galactosyltransferase activity of isolated saccular elements in response to in vitro trypsinization was similar to that observed in the rat liver Golgi apparatus. Electrophoresis and immunoblotting analyses revealed polypeptide modifications in the saccular elements during spermatozoa epididymal transit. In addition, a significant decline in the content of galactosyltransferase and sialyltransferase occurred in the saccular elements during spermatozoa transit from the caput to the cauda epididymides. The content of TGN-38, however, did not show any significant change, suggesting that the modification of the content of the saccular elements is selective. Considering the glycosylating ability of the saccular elements and that the diminution of glycosyltransferase activities in cytoplasmic droplets coincides with the maturation of spermatozoa during epididymal transit, we suggest a potential role for the saccular elements of cytoplasmic droplets in the maturation of spermatozoa during their transit through the epididymis.
\end{abstract}

\section{INTRODUCTION}

Spermatozoa released at spermiation from the seminiferous tubules of the testis are non-motile and infertile. It is only after their transit through the epididymal tract that motility and fertility are acquired. One of the most conspicuous morphological changes that occurs to spermatozoa during their transit through the epididymis is the displacement of the cytoplasmic droplets along their tail, and their subsequent detachment 
into the lumen of the corpus and the cauda epididymides (1). The cytoplasmic droplet (Fig. 1) is a small mass of cytoplasm that is retained by spermatozoa as they are released from the seminiferous epithelium of the testis during spermiation. Such a structure is ubiquitous to all mammalian species (2-4) and to some nonmammalian ones as well (5). The biological significance of this structure is unknown. At spermiation, the bulk of the late spermatid's cytoplasm, referred to as the residual body, is phagocytosed and disintegrated rapidly by the Sertoli cells lining the seminiferous epithelium (6). The segregation of the cytoplasmic droplet from the residual body appears to be specific, with the flattened membranous elements, termed saccular elements, being consistently retained in the cytoplasmic droplet but not in the residual body. Organelles, such as mitochondria, ribosomes, remnants of the endoplasmic reticulum, and lipid bodies, are eliminated via the residual body. This phenomenon not only suggests that phagocytosis of the residual body by the Sertoli cell involves a specific and selective mechanism, but also suggests that the saccular elements may have a biological function.

Infertility is often found to be caused by various genetic and environment-induced defects of the spermatozoa's tail. Barth and Oko provided evidence that many of these defects are associated with the trapping of the cytoplasmic droplet in the abnormal bend of the sperm tail (7). Studies have shown that ejaculates containing a high proportion of spermatozoa with attached droplets are correlated with altered epididymal function and reduced fertility (8-10), further suggesting a connection between the function of the cytoplasmic droplet and fertility. A recent study by Oko et al. revealed Golgi characteristics of the saccule elements (11). They further demonstrated the ability of the saccular elements to glycosylate endogenous proteins. These findings suggest that the saccular elements may have a potential role in modifying the plasma membrane of spermatozoa during epididymal transit.

In the present study, a comparison of the polypeptide composition and glycosyltransferase content was made between saccular elements of the cytoplasmic droplet isolated from either the caput or the cauda epididymal spermatozoa. In addition, the level of galactosyltransferase activity of the isolated saccular elements in response to trypsin digestion was analyzed. The significance of the modifications affecting protein distribution and enzyme activity in the saccular elements during epididymal transit is discussed in relation to spermatozoa maturation.

\section{METHODS}

\section{Isolation and Subfractionation of Cytoplasmic Droplets}

Nine adult male Sprague-Dawley rats (350-450 g; St. Constant, QC, Canada) were anesthetized with sodium pentobarbital (Somnitol, MTC Pharmaceuticals, Hamilton, ON, Canada). To clear any blood within the tissue, the epididymides of the animals were perfused with lactated Ringer's solution (Abbott laboratories Ltd., Montreal, QC, Canada) via the abdominal aorta. After trimming off the connective and adipose tissues, the epididymides were separated according to Reid and Cleland's criteria (12) into the caput (including the initial segment, the proximal caput, and the distal caput) and the cauda (including the proximal and distal cauda). All the subsequent procedures were performed separately for the caput and the cauda epididymides on ice or at 4deg.C. The tissues were rinsed in an STK buffer containing 0.25 M sucrose, $50 \mathrm{mM}$ Tris- $\mathrm{HCl}$ and $25 \mathrm{mM} \mathrm{KCl}$ (all chemicals used were from Sigma Chemical Co., St. Louis, MO, USA unless otherwise specified) at $\mathrm{pH}$ 7.4. Protease inhibitors, including phenylmethyl-sulfonyl fluoride at $1.0 \mathrm{mM}$ and aprotinin at 100 units/ml, were added to this buffer. For the galactosyltransferase biochemical assay with trypsinization, the protease inhibitors were omitted.

The isolation procedure elaborated here has been employed by our laboratory in other studies $(11,13)$. The epididymides were sliced into $2 \mathrm{~mm}$ sections in STK buffer. The minced tissue suspension was then vortexed lightly to facilitate the exudation of spermatozoa from the lumen of the epididymides. This suspension was 
then filtered through a 150 um Nitex netting (Thompson, Montreal, QC, Canada) to remove epithelial tissue. The sperm suspension was centrifuged at $3000 \mathrm{~g}$ for $15 \mathrm{~min}$ and the resulting sperm pellet was resuspended in $5.0 \mathrm{ml}$ of STK buffer. The supernatant was centrifuged at 80,000 $\mathrm{g}$ for 15 min to collect any freed cytoplasmic droplets into a pellet which was then resuspended together with the original pellet. The pellet suspensions were then passed through a 1.5 inch-long 20-gauge needle four times using a 10 cc syringe, which, as evaluated by phase-contrast microscopy, detached the cytoplasmic droplets that were still retained on the sperm tails. The resulting homogenate was centrifuged at $150 \mathrm{~g}$ for $10 \mathrm{~min}$ in order to obtain a pellet fraction $(\mathrm{P})$ and to float the detached cytoplasmic droplets into the supernatant $(\mathrm{S})$. The supernatant was loaded onto a four-step sucrose discontinuous gradient $(0.6 \mathrm{M}, 0.8 \mathrm{M}, 1.0 \mathrm{M}, 1.2 \mathrm{M})$ prepared in $50 \mathrm{mM}$ Tris$\mathrm{HCl}$ and $25 \mathrm{mM} \mathrm{KCl}$ buffer, $\mathrm{pH} 7.4$, and centrifuged at 200,000 g for 1.5 hrs using a Beckman SW 41-Ti rotor. Each of the cellular fractions collected from the five interfaces of the gradient was analyzed by electron microscopy and electrophoresis. Each was also assayed for protein concentration, galactosyltransferase activity, and sialyltransferase activity.

\section{Electron Microscopy with Epon Embedded Sections}

Cellular fractions collected from each interface of the discontinuous gradient were fixed overnight at 4deg.C with $2.5 \%$ glutaraldehyde (M.E.C.A. Ltd., Montreal, QC, Canada) buffered in $0.1 \mathrm{M}$ sodium cacodylate (pH 7.4) (M.E.C.A Ltd.) containing $0.05 \% \mathrm{CaCl}$. Fixed pellets were then submersed in $2 \%$ type IX agarose, allowed to solidify, and subsequently post-fixed with $1.5 \%$ potassium ferrocyanide (Fisher Scientific Co., Fair Lawn, NJ, USA) reduced 1.0\% osmium tetroxide (M.E.C.A. Ltd.) (14) for $1 \mathrm{hr}$ at 4deg.C. Each pellet was dehydrated in ethanol and propylene oxide, and embedded in Epon 812 (M.E.C.A. Ltd.). Thin tissue sections were mounted on 300 mesh uncoated copper grids and stained with $4 \%$ uranyl acetate (M.E.C.A. Ltd.) for 5 min and with $6 \%$ lead citrate (M.E.C.A. Ltd.) for 2 min $(15,16)$. The ultrathin sections were examined under a Philips 400 electron microscope.

\section{Analysis of Polypeptide Compositions}

\section{Sodium Dodecyl Sulfate Polyacrylamide Gel Electrophoresis (SDS-Page)}

Cellular fractions collected from each interface of the discontinuous gradient and from the supernatant and pellet of the first centrifugation were solubilized in a solution of $2 \%$ sodium dodecyl sulfate (SDS) (Mallinckrodt Canada Inc., Pointe-Claire, QC, Canada) and 5\% b-mercaptoethanol for 5 min at 100deg.C. Samples were then loaded on linear gradient (8-18\%) SDS-discontinuous polyacrylamide gels according to the procedure described by Laemmli (17). For the purpose of comparison among different cellular fractions, the quantity of each sample was normalized to 20 or $40 \mathrm{ug}$ of protein. Apparent molecular masses were determined from the mobility of low molecular weight standards (Pharmacia low molecular weight protein standards, Piscataway, NJ). The gels were stained with Coomassie Brilliant Blue (Bio-rad, Richmond, CA).

\section{Western Blotting}

Polypeptides $(300 \mathrm{ug} / \mathrm{gel})$, from the gels of fraction 3 isolated from the caput and the cauda epididymides, were transferred on to immobilon paper (Immobilon-P transfer membranes, Millipore, Mississauga, ON, Canada) using a Hoefer Transpher Apparatus (Hoefer Scientific Instruments, San Francisco, CA, USA) according to the techniques of Towbin et al. (18) and Towbin and Gordon (19). Electrophoretic transfer was carried out at 0.5-0.6 A for 3-5 hrs. Following transfer, the blots were washed three times at room temperature each time for $15 \mathrm{~min}$ in $10 \mathrm{mM}$ Tris- $\mathrm{HCl}, 0.9 \% \mathrm{NaCl}$, and $0.5 \%$ Tween-20, $\mathrm{pH}$ 7.4. Immobilon blots were stained with Coomassie Brilliant Blue. The washed immobilon strips transferred with the polypeptide were first blocked with 5\% goat serum in TWBS $(20 \mathrm{mM}$ Tris-HCl buffer with $0.9 \%$ saline and $0.1 \%$ Tween-20) for $2 \mathrm{hrs}$ at room temperature. The strips were then incubated for $2 \mathrm{hrs}$ at room temperature 
with rabbit polyclonal antibody raised against the saccular elements of the cytoplasmic droplet (anti-SE) (11). Antibodies were diluted in TWBS with $0.1 \%$ bovine serum albumin (BSA) and $1 \%$ goat serum. The dilutions used were determined empirically and reflected the optimum antibody concentration that minimized nonspecific labeling. The strips were subjected to four 5-min rinses in TWBS, followed by a 30-min wash with $5 \%$ goat serum in TWBS. Following a $2 \mathrm{hr}$ incubation at 37deg.C in a 1:1000 dilution of alkaline phosphatase-conjugated $\mathrm{F}(\mathrm{abl}) 2$ goat anti-rabbit immunoglobulin $\mathrm{G}$ (Cappel- Cooper Biomedical Inc., Malvern, PA, USA), the strips were washed at $\mathrm{pH} 9.6$ with TWBS and $50 \mathrm{mM}$ sodium glycinate with $0.5 \%$ Tween-20. The phosphatase reaction was developed according to McGadey (20) by incubating blots for 10$20 \mathrm{~min}$ in a solution composed of $0.01 \%$ Nitro blue tetrazolium, $0.005 \%$-bromo- 4-chloro-3-indolyl phosphate, and $4 \mathrm{mM}$ of $\mathrm{MgCl} 2$ of in a $50 \mathrm{mM}$ sodium glycinate solution for 10-20 min. Controls consisted of replacing anti-SE antibodies with preimmune serum, anti-actin antibodies (21), or anti-tubulin antibodies (22).

\section{Enzyme Assays of Cellular Fractions}

\section{Galactosyltransferase Assay}

Galactosyltransferase (UDP-Gal: 2-acetamido-2-de-oxy-D-glucosyl-glycopeptide galactosyltransferase) activity in the cellular fractions obtained from the discontinuous gradient were assayed using a modified technique used for Golgi apparatus $(23,24)$. This technique is based on the transfer of uridine diphosphate galactose (UDP-[3H] galactose [galactose-1-3H(N)]-, Du Pont Inc., Markham, ON, Canada) to the exogenous acceptor, ovomucoid. The donor sugar consisted of UDP-[3H] galactose that was dried with nitrogen and resuspended in UDP-galactose (Uridine-5' Diphospho-galactose, sodium salt) to a specific activity of $0.045 \mathrm{uCi} / \mathrm{nmol}$. The incubation mixture contained $0.0441 \mathrm{mM}$ UDP-galactose $(0.2 \mathrm{uCi}), 30 \mathrm{mM}$ sodium cacodylate buffer ( $\mathrm{pH}$ 6.5), $2.0 \mathrm{mM}$ adenosine triphosphate (disodium salt), $30 \mathrm{mM} \mathrm{MnCl} 2,30 \mathrm{mM}$ b-mercaptoethanol, $0.2 \%(\mathrm{v} / \mathrm{v})$ Triton X-100, $4.0 \mathrm{mg} / \mathrm{ml}$ ovomucoid, and $30 \mathrm{ul}$ of the cell fraction sample (5$20 \mathrm{ug}$ protein), giving a final volume of $100 \mathrm{ul}$. Incubations were carried out for $15 \mathrm{~min}$ at 37[[ring]]C and stopped by the addition of $1.0 \mathrm{ml}$ of ice-cold 1\% phosphotungstic acid (J.T. Baker Inc., Phillipsburg, NJ, USA) in $0.5 \mathrm{~N} \mathrm{HCl}$. The mixtures were kept on ice for $30 \mathrm{~min}$ to allow precipitation of the protein and then

pelleted at 13,000 r.p.m. by micro-centrifugation. The pellets were resuspended and repelleted twice in $1.0 \%$ phosphotungstic acid. After overnight drying, the pellets were dissolved in $0.5 \mathrm{ml}$ of $1.0 \mathrm{~N}$ sodium hydroxide, incubated at $65 \mathrm{deg} . \mathrm{C}$ for $1 \mathrm{hr}$, and a portion of each sample (100 ul) was mixed with $5.0 \mathrm{ml}$ of scintillation cocktail (Opti-flour, Packard Instrument Co., Downer Grove, IL, USA). The radioactive content of the scintillation mix was determined in a Packard Spectrometer model 460 (Packard Instrument Co.) and cpm converted to dpm by the channels-ratio and external standard methods (25).

The effect of trypsin on galactosyltransferase activity was studied as follows: $70 \mathrm{ul}$ (20-30 ug protein) of fraction 3 (interface between $0.8 \mathrm{M}$ and $1.0 \mathrm{M}$ sucrose buffer) obtained from the caput epididymal spermatozoa was incubated for $1 \mathrm{hr}$ at $0 \mathrm{deg}$.C with trypsin (type IX from porcine pancreas) at different concentrations $(0.1 \mathrm{ug} / \mathrm{ml}, 1 \mathrm{ug} / \mathrm{ml}, 10 \mathrm{ug} / \mathrm{ml}, 50 \mathrm{ug} / \mathrm{ml}$, and $100 \mathrm{ug} / \mathrm{ml})$ in $50 \mathrm{mM}$ Hepes buffer with $5 \mathrm{mM}$ $\mathrm{KCl}, 1 \mathrm{mM} \mathrm{CaCl} 2$, and $1 \mathrm{mM} \mathrm{MgCl} 2$, in the presence or absence of $0.1 \%(\mathrm{v} / \mathrm{v})$ Triton X-100. The galactosyltransferase assay was performed as described above and the enzyme activity of each trypsinized sample was expressed as a percentage of the total enzyme activity of a control sample without trypsinization.

\section{Sialyltransferase Assay}

The assay for sialyltransferase (CMP-N-acetyl-neuraminate: D-galactosyl-glycoprotein N-acetylneuraminyltransferase), was modified from Bretz et al. (26). The donor sugar consisted of CMP-[3H] N-acetylneuraminic acid (NANA) (Cytidine 5'- monophosphate sialic acid, [sialic-9-3H]-, Du Pont Inc., Markham, ON, Canada) that was dried and resuspended in CMP-NANA (Cytidine 5'-monophospho- (NANA), ammonium salt) to a specific activity of $4.3 \mathrm{uCi} / \mathrm{mmol}$. The incubation mixture contained $1.0 \mathrm{mM}$ of CMP- 
NANA (0.43 uCi), $100 \mathrm{mM}$ cacodylate buffer (pH 5.8), $40 \mathrm{mM}$ b-mercaptoethanol, $0.4 \%$ (v/v) Triton X-100, $17.5 \mathrm{mg} / \mathrm{ml}$ asialofetuin (Sigma Chemical Co.) as an exogenous acceptor for sialyltransferase, and $60 \mathrm{ul}$ of the cell fraction sample (10-40 ug protein) giving a final volume of $100 \mathrm{ul}$. Incubations were carried out for 30 min under identical conditions as in the galactosyltransferase assay. The washing and counting procedures were also identical to those used in the galactosyltransferase assay.

\section{Immunocytochemistry of Cellular Fractions and Epididymal Tissue}

The anti-b-1,4-galactosyltransferase, the antiserum N7 raised to native human milk galactosyltransferase (27), was obtained from Dr. E. Berger (Physiologishes Institut, Zurich, Switzerland). The anti-a-2,6sialyltransferase, the IgG purified from bacterially expressed sialyltransferase (28), was obtained from Dr. J. Paulson (Cytel Corp., San Diego, CA, USA). The anti-TGN38 antiserum was obtained from K. Howell (University of Colorado, Denver, CO, USA) and has been described and characterized by Luzio et al. (29). The dilutions used reflected the optimum concentrations and labeling for each antibody preparation that minimized non-specific labeling.

\section{Light Microscopy}

Rats used for light microscopy studies were fixed by perfusion through the abdominal aorta with Bouin's fixative for $10 \mathrm{~min}$ at room temperature. The epididymides were removed and immersed in the same fixative overnight, followed by several washes with $70 \%$ ethanol. The tissue was dehydrated with ethanol and embedded in paraffin for routine histological sections. Paraffin sections of 5 um thickness were cut and mounted onto glass slides. The tissue sections were deparaffinized with xylene (Fisher Scientific Co.) and hydrated in graded ethanol solutions. During hydration, the sections were incubated in $70 \%$ ethanol containing $1 \%$ lithium carbonate for $5 \mathrm{~min}$ to inactivate any residual picric acid remaining from the fixation procedure. To eliminate any endogenous peroxidase activity, the sections were also incubated for $5 \mathrm{~min}$ in ethanol containing $1 \%(\mathrm{v} / \mathrm{v})$ hydrogen peroxide. Following hydration, the sections were first washed in a 300 $\mathrm{mM}$ glycine solution to block any free aldehyde groups, and then briefly washed in $20 \mathrm{mM}$ Tris- $\mathrm{HCl}$ buffer with $0.9 \%$ saline at $\mathrm{pH} 7.4$ (TBS) containing $0.1 \%$ (w/v) bovine serum albumin (BSA).

For immunolabeling, the sections were first blocked for $15 \mathrm{~min}$ in TBS containing $10 \%$ goat serum. They were then incubated for $1 \mathrm{hr}$ with primary antibodies at various dilutions in TBS. The sections were washed four times, 5 min each, with TBS containing $0.1 \%$ Tween-20. They were then blocked with $10 \%$ goat serum in TBS for $15 \mathrm{~min}$, and incubated for $1 \mathrm{hr}$ with goat anti-rabbit IgG conjugated to peroxidase at a dilution of 1:250 in TBS. All sections were then washed thoroughly in TBS containing $0.1 \%$ Tween-20. The immunoperoxidase reaction was developed by incubating the sections for $10 \mathrm{~min}$ with $0.03 \%$ hydrogen peroxide and $0.05 \%$ 3,3'-diaminobenzidine tetrahydrochloride in TBS containing $0.01 \mathrm{M}$ imidazole at $\mathrm{pH} 7.6$ (30). This permitted the deposition of an insoluble brown polymer at the site of the antigen-antibody reaction (31). After washing, the sections were counterstained with $0.1 \%$ methylene blue (Aldrich Chemical Co., Milwaukee, WI, USA), dehydrated in an ethanol gradient, immersed in xylene, and mounted in Permount (Fisher Scientific Co.). Affinity purified antibodies against a host of other membranous proteins, including insulin receptors and epidermal growth factor receptors, were used as controls.

\section{Ultrathin Frozen Sections}

Cellular fractions were fixed with $4 \%$ paraformaldehyde (Fisher Scientific Co.) in 0.1 M sodium cacodylate buffer ( $\mathrm{pH} 7.4)$ with $0.1 \% \mathrm{CaCl} 2(\mathrm{w} / \mathrm{v})$ for $1 \mathrm{hr}$ at $4 \mathrm{deg}$.C. The fixed suspensions were then embedded in $2 \%$ type IX agarose, allowed to solidify, washed twice with $0.1 \mathrm{M}$ cacodylate buffer containing $7 \%$ sucrose, and equilibrated for a minimum of $30 \mathrm{~min}$ with $2.3 \mathrm{M}$ sucrose (32). The pellets were frozen directly in liquid 
nitrogen-cooled propane and stored in liquid nitrogen (Linde Union Carbide, Toronto, ON, Canada). All the subsequent steps were based on the frozen section procedures developed by Tokuyasu $(32,33)$, Griffiths et al. (34), and Geuze et al. (35). Thin sections were mounted on carbon-coated copper grids (200 mesh) with a formvar film. Each grid was then kept at room temperature on a drop of 5\% fetal calf serum (FCS) (Gibco Laboratories, Grand Island, NY, USA) in $20 \mathrm{mM}$ Tris-HCl buffer and TBS containing $0.1 \%(\mathrm{w} / \mathrm{v})$ BSA.

For immunolabeling, the grids were first incubated for 15 min with a blocking reagent in TBS containing $0.1 \%$ bovine serum and $10 \%$ FCS. This was followed by incubation with the primary antibodies at their optimal dilution. Following three 5-min washes with $1 \%$ FCS in TBS, the grids were again incubated with the blocking reagent for $15 \mathrm{~min}$. The grids were then incubated on drops of colloidal gold $(8 \mathrm{~nm})$-conjugated goat anti-rabbit IgG (Zollinger Inc., Montreal, QC, Canada) at a dilution of 1:20 followed by three 5-min washes in TBS and two 5-min washes in $\mathrm{ddH} 2 \mathrm{O}$. Controls used were identical to those mentioned above.

The staining and embedding procedures described by Tokuyasu (32) were used. The grids containing the sections were stained with $2 \%$ aqueous uranyl acetate which had been adjusted to $\mathrm{pH} 7.3$ with oxalic acid and ammonium hydroxide. Finally, the grids were coated with $0.5 \%$ methyl cellulose (Lab Grade Ac5900, Anachemia Canada Inc., Montreal, QC, Canada) containing 0.4\% aqueous uranyl acetate and air dried.

\section{Statistical Analysis}

The results of enzyme specific activities of each subfraction in the biochemical assays were analyzed by single factor analysis of variance test (ANOVA) and Student Newman Keuls (SNK) test to detect any significant difference between each fraction. A two-tailed paired Student's t test was used to detect any significant difference between the enzyme specific activities of the corresponding subfractions that were isolated from the caput and the cauda epididymal spermatozoa.

\section{RESULTS}

\section{Isolation of Saccular Elements of Cytoplasmic Droplets}

Saccular membranous elements of cytoplasmic droplets were isolated from spermatozoa obtained from both the caput (including initial segment, proximal, and distal caput) and the cauda (including proximal and distal cauda) epididymides. Analysis by phase-contrast and electron microscopy revealed that the corresponding fractions obtained from spermatozoa of the caput and the cauda epididymides were morphologically identical. Thus the morphological description below applies to isolations from both regions of the epididymides. To ensure that the cytoplasmic droplets were detached from the spermatozoa, the initial sperm suspension was subjected to a shear force by passing it several times through a 20 gauge needle. The suspension was then centrifuged at low speed into a supernatant (S) fraction, containing mostly cytoplasmic droplets, as determined by electron microscopy (Fig. 2), and a pellet (P) fraction containing spermatozoa (Fig. 3). The supernatant represents the fraction enriched in cytoplasmic droplets. It is important to note, however, that a substantial number of cytoplasmic droplets were still found in the pellet. The cytoplasmic droplet-enriched supernatant was subsequently used to subfractionate the droplets on a four-step sucrose discontinuous gradient. By ultrastructural analysis of the five resulting fractions, it was found that the saccular elements in question resided mainly in fractions 3 and 4 of the gradient.

Fraction 3 of the gradient corresponded to the interface between $0.8 \mathrm{M}$ and $1.0 \mathrm{M}$ sucrose buffer (specific gravity: 1.105 and 1.130, respectively). It was comprised of numerous plasma membrane-enclosed droplets containing flattened, straight, and C-shaped saccular elements (Fig. 4), resembling those found within droplets in situ, except for a loss of cytoplasm. With the exception of the plasma membrane found surrounding these discrete packets of saccules, no significant contamination by other sperm components was 
noted. Fraction 4 of the gradient corresponded to the interface between $0.6 \mathrm{M}$ and $0.8 \mathrm{M}$ sucrose buffer (specific gravity: 1.080 and 1.105, respectively). It was also characterized by saccular membranous elements (Fig. 5) similar in size and shape to those described above for fraction 3. However, in contrast to fraction 3 , the saccular elements in fraction 4 were not found in discrete packets enclosed by a plasma membrane, but were loosely arranged throughout the fraction.

EM immunocytochemistry on frozen sections using specific antisera against rat cytoplasmic droplet saccular elements (11) revealed strong immune reaction to the isolated saccular elements from fraction 3 (Fig. 6). The intensity of the immune reaction did not differ significantly between fraction 3 isolated from the caput and fraction 3 isolated from the cauda epididymal spermatozoa.

Although some saccular elements were found in the other three interfaces of the discontinuous sucrose gradient (i.e., fraction 1 or the pellet fraction, fraction 2 from the $1.2 \mathrm{M} / 1.0 \mathrm{M}$ sucrose gradient interface, and fraction 5 from the $0.6 \mathrm{M} / 0.25 \mathrm{M}$ sucrose gradient interface), these elements did not predominate as in fractions 3 and 4, but were intermixed with other sperm fragments and components.

\section{Polypeptide Profiles of the Saccular Elements}

Polypeptide compositions of various isolated subcellular fractions from spermatozoa of the initial segment and the caput epididymides were analyzed on 8-18\% polyacrylamide gradient gels (Fig. 7). Fraction S (Fig. 7, lane S), which represents a crude extract of cytoplasmic droplets, contains numerous polypeptide bands in the range of 15 to $100 \mathrm{kDa}$. Major peptide bands were observed at 21, 41, 44, 68, 75, and $80 \mathrm{kDa}$.

Subfractionation of fraction $\mathrm{S}$ yielded fractions 1 to 5 and the residual fraction $(\mathrm{R})$, which is composed of the contents of the leftover sucrose gradient (Fig. 17, lanes 1 to 5 and R). Fraction 3 (lane 3), where saccular elements of cytoplasmic droplets were observed by electron microscopy, was composed of numerous polypeptides. Seven of these were most prominent, having molecular masses of 33, 36, 44, 68, 75, 97, and $110 \mathrm{kDa}$. These seven polypeptides, except for the $68 \mathrm{kDa}$ one, were more prominent than in the original crude extract (fraction S). Thus, this represents an enrichment of these polypeptides in the isolated saccular elements. Note also that the distribution of polypeptides in fractions 2, 3, and 4 was similar.

The polypeptide band pattern of isolated saccular elements from fraction 3 that were immuno-blotted (Fig. 8, lane $\mathrm{H}$ ) were comparable to those found on polyacrylamide gels (Fig. 7, lane 3). The polypeptide composition of fraction 3 isolated from the caput epididymal spermatzoa (Fig. 8, lane H) was distinct from that isolated from the cauda epididymal spermatozoa (Fig. 8, lane T). On immunoblots, the antisera raised against isolated saccular elements from spermatozoa of the entire epididymis (11) bound most strongly to polypeptides of the caput saccular elements of molecular masses of 17, 36, and $44 \mathrm{kDa}$ (Fig. 8, lane H3). In comparison, immunoblots of the cauda saccular elements (Fig. 8, lane T3) probed with the same anti-serum showed numerous additional polypeptide immunoreactions between 30 to $94 \mathrm{kDa}$.

\section{Subcellular Distribution of Golgi Apparatus Marker Enzymes in Epididymal Spermatozoa}

The enzyme activities of subcellular fractions and residues obtained from the caput and the cauda spermatozoa are presented according to de Duve et al. (36) (Fig. 9A and 9B). The highest galactosyltransferase and sialyltransferase specific activities were found in fraction $3(\mathrm{p}<0.005)$ of the caput spermatozoa and represent an enrichment of 6.4 and 4.7 fold, respectively, over the crude spermatozoa supernatant (fraction S). The exact percentage of recovery of the protein content and enzyme activities for the various fractions relative to the whole supernatant fraction is presented in Tables 1 and 2 . The absolute values for the specific activities of galactosyltransferase and sialyltransferase in fraction 3 were $3.54+/-0.47$ $\mathrm{nmol} / \mathrm{min} / \mathrm{mg}$ protein and $1.36+/-0.15 \mathrm{nmol} / \mathrm{min} / \mathrm{mg}$, respectively. In contrast to the caput epididymal sample, no significant enrichment of the Golgi marker enzyme activities was found in any of the fractions 
from the cauda epididymal sample (Table 3 and Fig. 9B). In fact, the absolute specific activities of the two Golgi marker enzymes in the saccular element fractions 3 and 4 of the cauda epididymal spermatozoa were found to be more than 10 times lower than that of the corresponding fractions in the caput epididymal spermatozoa $(\mathrm{p}<0.001)$.

The effect of trypsinization on galactosyltransferase activity residing in the saccular elements is shown in Fig. 10. With Triton X-100 present, the enzyme activity declined sharply at levels of trypsin ranging from 0 to 1 $\mathrm{ug} / \mathrm{ml}$. In this range of trypsin, no significant reduction of galactosyltransferase activity occurred under incubation conditions free of Triton X-100. When trypsin levels were increased to $100 \mathrm{ug} / \mathrm{ml}$ under incubation periods free of Triton X-100, the transferase activity was maintained at $55 \%$ of control values.

\section{Immunocytochemical Detection of Golgi Apparatus Markers in the Epididymis}

\section{Light Microscopy}

Paraffin sections through the initial segment of the epididymis probed with anti-serum against the trans Golgi network protein (TGN-38) revealed intense immunoperoxidase staining over the supranuclear region of the epithelial principal cells lining the tubules (Fig. 11). The reaction in the principal cells, discernible as a dense anastomotic network localized in the supranuclear region, is believed to correspond to the region of the Golgi apparatus. No reaction was evident over the remainder of the cytoplasm or the nucleus of the cell. In the tubular lumen, the immunoreaction was localized over discrete bodies located along the sperm tail. These bodies correspond to the cytoplasmic droplets (Fig. 11). No staining was detectable over other regions of the tail or the head of the spermatozoa. The TGN-38 labeling pattern described above was characteristic of all other regions of the epididymis (Fig. 12).

Anti-b-1,4-galactosyltransferase serum revealed an intense reaction over the supranuclear region of the epithelial principal cells in the initial segment of the epididymis (Fig. 13). This reaction was similar to that shown for anti-TGN-38. As seen in the lumen, an intense reaction was also observed in the sperm tail over discrete bodies corresponding to the cytoplasmic droplets. However, in contrast to the strong anti-TGN-38 reaction observed over the cytoplasmic droplets throughout the epididymis, the anti-galactosyltransferase reaction was undetectable in the cytoplasmic droplets of the corpus and the cauda epididymides. It was undetectable even though it remained present over the supranuclear region of the principal cells (Fig. 14).

Furthermore, anti-a-2,6-sialyltransferase antibodies reacted exclusively with the supranuclear region of the principal cell and with the cytoplasmic droplets within the initial segment and the caput epididymides (Fig. 15). However, as with the anti-galactosyltransferase reaction, little or no labeling of the cytoplasmic droplets was found in the corpus and the cauda epididymides (Fig. 16). Controls, using non-immune rabbit sera and affinity purified antibodies against epidermal growth factor receptor and insulin receptor, did not show any reaction over the epithelium or the spermatozoa in the epididymis.

\section{Electron Microscopy}

Ultrathin frozen sections of isolated saccular elements (fraction 3), obtained from the initial segment and the caput spermatozoa, labeled intensely with anti-galactosyltransferase serum (Fig. 17). Isolated saccular elements obtained from the cauda spermatozoa labeled weakly with anti-galactosyltransferase serum (Fig. 18), which corresponds with our results from LM immunocytochemistry. This tissue pattern of immunogold labeling was also found with anti-sialyltransferase serum.

\section{DISCUSSION}




\section{Isolation of the Saccular Elements of Cytoplasmic Droplets}

Several protocols for the isolation of cytoplasmic droplets of spermatozoa have been developed (37-43). However, our method is the first to isolate the saccular elements of the cytoplasmic droplets. An essential feature of our isolation protocol is the use of a multi-step sucrose gradient which allows for the separation of various cellular and subcellular components of the crude cytoplasmic droplet fraction. The gradation in buoyant density during ultra-centrifugation avoids abrupt changes in the centrifugal forces and thus reduces the shearing forces on the cellular components' sediment. Furthermore, as sedimentation occurs on sucrose interfaces, harsh pelleting of subcellular fractions against the tube wall is avoided. This may allow better preservation of the morphology and integrity of the subcellular structures for further analysis. The purity of the isolated saccular elements, as examined by electron microscopy, showed minimal contamination with other visible cellular structures. Polyclonal antibodies raised against isolated saccular elements (11), which reacted specifically with saccular elements in situ, confirmed the identity of our isolated fraction. The buoyant density of the sucrose interfaces, where the saccular elements were isolated, were 1.1302/1.1054 (fraction 3) and 1.1054/1.0797 (fraction 4). These values correspond to that of heavy and light Golgi subfractions, respectively, from rat hepatocytes (44). This finding is consistent with the proposal that the saccular elements of cytoplasmic droplets are Golgi-derived (45).

\section{Distribution of Golgi Apparatus Marker Enzyme Activities in the Saccular Elements of Cytoplasmic Droplets}

Using radioactive glycosylation assays, we confirmed the localization of galactosyltransferase and sialyltransferase, two well-known marker enzymes of the Golgi apparatus (46-49), in the isolated fractions of saccular elements from the caput epididymal spermatozoa $(11,13)$. The specific activities of galactosyltransferase and sialyltransferase, measured by exogenous glycosylation, have been reported for the Golgi apparatus using different incubation media and conditions. For galactosyltransferase, the reported specific activity in the rat liver Golgi apparatus ranges from 3.5 to $13 \mathrm{nmol} / \mathrm{mg}$ protein/min $(26,44,50,51)$. For sialyltransferase, the reported specific activity ranges from 8 to $14 \mathrm{nmol} / \mathrm{mg}$ protein/min (26). The measured galactosyltransferase and sialyltransferase activities of isolated saccular element fractions in this study were 2.1 and $1.1 \mathrm{nmol} / \mathrm{mg}$ protein/min, respectively. Although the absolute specific enzyme activities of the two Golgi marker enzymes in the saccular elements were lower, the values approximate those reported in the literature for the rat liver Golgi apparatus.

Galactosyltransferase in the saccular element fraction is resistant to trypsinization in the absence of the detergent Triton X-100, which suggests that the enzyme is localized internally. With $10 \mathrm{ug} / \mathrm{ml}$ of trypsin, the initial drop in enzyme activity to around $60 \%$ of control values is probably due to trypsin inactivation of free galactosyltransferase present in a soluble form in the subfraction. Thereafter, despite the increase in trypsin levels, the enzyme activity does not significantly decrease any further. A similar pattern of response of galactosyltransferase activity to various trypsin concentrations is also observed in the rat liver. 